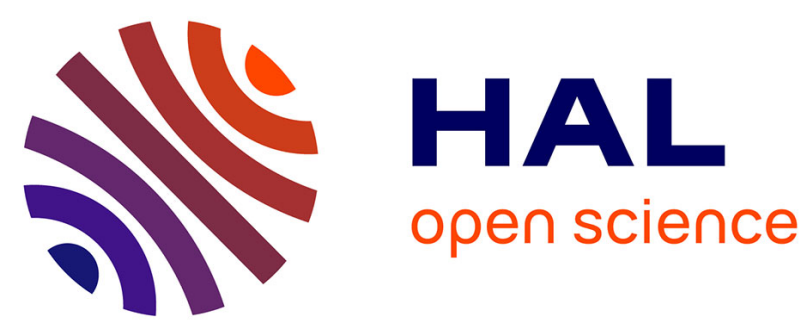

\title{
Multidrug efflux pumps and their role in antibiotic and antiseptic resistance: a pharmacodynamic perspective
}

Sandrine Alibert, Joannah N 'Gompaza Diarra, Jessica Hernandez, Aurélien

Stutzmann, Marwa Fouad, Gérard Boyer, Jean-Marie Pagès

\section{To cite this version:}

Sandrine Alibert, Joannah N 'Gompaza Diarra, Jessica Hernandez, Aurélien Stutzmann, Marwa Fouad, et al.. Multidrug efflux pumps and their role in antibiotic and antiseptic resistance: a pharmacodynamic perspective. Expert Opinion on Drug Metabolism and Toxicology, 2016, 13 (3), pp.301-309. 10.1080/17425255.2017.1251581 . hal-01425015

\section{HAL Id: hal-01425015 \\ https://hal-amu.archives-ouvertes.fr/hal-01425015}

Submitted on 16 Feb 2017

HAL is a multi-disciplinary open access archive for the deposit and dissemination of scientific research documents, whether they are published or not. The documents may come from teaching and research institutions in France or abroad, or from public or private research centers.
L'archive ouverte pluridisciplinaire HAL, est destinée au dépôt et à la diffusion de documents scientifiques de niveau recherche, publiés ou non, émanant des établissements d'enseignement et de recherche français ou étrangers, des laboratoires publics ou privés. 


\title{
Expert Opinion on Drug Metabolism \& Toxicology
}

\section{Multidrug efflux pumps and their role in antibiotic and antiseptic resistance: a pharmacodynamic perspective}

\author{
Sandrine Alibert ${ }^{\mathrm{a}}$, Joannah N'gompaza Diarra ${ }^{\mathrm{a}}$, Jessica Hernandez ${ }^{\mathrm{a}}$, Aurélien Stutzmann ${ }^{\mathrm{a}}$, Marwa Fouad ${ }^{\mathrm{b}}$, \\ Gérard Boyer ${ }^{\mathrm{a}}$ and Jean-Marie Pagès ${ }^{\mathrm{a}}$
}

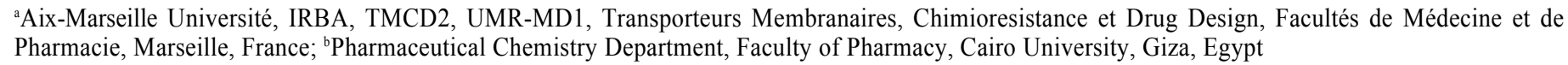

CONTACT Sandrine Alibert ～sandrine.alibert@univ-amu.fr ～Aix-Marseille Université, UMR-MD1, Faculté de Pharmacie, Département de Chimie Pharmaceutique, Service de Chimie Thérapeutique \& Minérale, 27 boulevard Jean Moulin 13385, Marseille cedex 05, France

\begin{abstract}
Introduction: Worrying levels of bacterial resistance have been reported worldwide involving the failure of many available antibiotic treatments. Multidrug resistance (MDR) in Gram-negative bacteria is often ascribed to the presence of multiple and different resistance mechanisms in the same strain. RND efflux pumps play a major role and are an attractive target to discover new antibacterial drugs. Areas covered: This review discusses the prevalence of efflux pumps, their overexpression in clinical scenarios, their polyselectivity, their effect on the intracellular concentrations of various antibiotics associated with the alteration of the membrane permeability and their involvement in pathogenicity are discussed.

Expert opinion: Efflux pumps are new targets for the development of adjuvant in antibiotic treatments by of efflux pump inhibition. They may allow us to rejuvenate old antibiotics acting on their concen- tration inside the bacteria and thus potentiating their activity while blocking the release of virulence factors. It is a pharmacodynamic challenge to finalize new combined therapy.

KEYWORDS

Efflux pump; Gram-negative bacteria; antibiotic; multidrug resistance; inhibitor

Article highlights

- Efflux pumps play a major role in multidrug resistance in Gram- negative bacteria

- The pharmacodynamic role of RND efflux pumps in antibiotic resis- tance opens up new perspectives to alternative therapeutics

- Targeting efflux pumps consists in solving the problems of polyse- lectivity, drug accumulation, membrane permeability and pathogeni- city related to bacterial resistance mechanisms

- How to validate efflux pumps as target for new combined therapy?

- Efflux pump inhibitor could be used as adjuvant in antibiotic treatments
\end{abstract}

\section{Introduction}

For more than half a century, antibacterial drugs including antibiotics and biocides from natural or chemical origin have been considered as a magic bullet to cure infectious diseases. Already in 1945, after the discovery of penicillin, Alexander Fleming advised that bacteria could become resistant to this pioneer class of $\beta$-lactam drugs. In 2014, WHO reports [1] worrying levels of bacteria resistance worldwide involving the failure of many available antibiotic treatments for common and life-threatening infections acquired in hospitals and in the community. Indeed, the large use (human and veterinary) of new antibacterial drugs has strongly supported the emergence of resistance mechanisms to protect bacteria. The spreading of resistance is a natural advancing response for microorganisms, but it is increased by the continuous pressure exerted by widespread and misuse of antibiotics [2-6]. Moreover, antibacterial resistance has serious consequences on outcomes for patients and healthcare spending. Urgency is intensified by the lack of new therapeutics in research and development pipeline particularly for the treatment of gramnegative bacteria (e.g. intestinal bacteria such as Escherichia coli and Klebsiella, and environmental opportunistic bacteria such as Pseudomonas and Acinetobacter) infections (belonging to the ESKAPE group $[7,8])$.

Resistance to antibiotics is multifaceted and multifactorial. Indeed, multidrug resistance (MDR) in gram-negative bacteria is often ascribed to the presence of multiple and different resistance mechanisms expressed in the same strain and active against various antibiotic families. The MDR phenotype has been attributed to both acquired and intrinsic mechanisms of resistance. However, the resistance-nodulation-division (RND) efflux pumps in gram-negative bacteria play a major role in MDR, as the pathogen survival is dependent not only on enzymes to inactivate specific drugs but also on efflux to export them $[9,10]$. The expression of an efflux pump, together with the outer membrane barrier, contributes to a severe decrease in intracellular concentrations of various antibiotic classes and consequently in its reduced activity in gram-negative MDR strains [9]. Efflux also affects biocidal agents comprising disinfectants, antiseptics, and preservatives that are commonly practiced in medicine [11,12]. Moreover, it is also involved in bacterial colonization and virulence $[10,13-16]$ and contributes to the acquisition of additional mechanisms of resistance that includes the mutation in antibiotic targets (e.g. mutation in gyrase/topoisomerase for quinolone) or the production of enzymes that degrade antibiotics (e.g. $\beta$-lactamases). In clinical isolates, efflux is also observed in association with the alteration of the outer membrane permeability $[17,18]$ (e.g. loss of outer membrane porin channels).

The polyspecificity of efflux pumps makes difficult the identification of precise pharmacophoric groups at the drug surface involved in the recognition and transport by these pumps. However, efflux pumps represent attractive target in order to restore the intracellular concentration of antibacterial agents [19-22]. To rejuvenate the activity of old antibiotics by 
targeting resistance mechanisms in clinical resistant isolates, these drugs can be combined with adjuvant molecules such as chemosensitizers (e.g. membrane permeabilizer or efflux inhibitor [19,23]). As the counteraction of influx and efflux routes finally determines the effective concentration of drugs inside bacterial cells, targeting membrane transporters could support a winning pharmacodynamic perspective against antibiotic resistance.

\section{Efflux pumps-based MDR in gram-negative bacteria}

In resistant clinical isolates of gram-negative bacteria, the widespread drug active transporter system is the AcrAB-TolC/ MexAB-OprM efflux pumps [9,10,24]. These tripartite efflux structures were first identified and characterized in $E$. coli and $P$. aeruginosa strains $[25,26]$. Advances in biochemistry and molecular biology enabled the discovery of numerous related RND pumps in nearly all clinically important bacteria [27-30].

These efflux pumps promote the extrusion of a large diversity of compounds from the cell including disinfectants, dyes, detergents, organic solvents [31], and structurally unrelated antimicrobial agents [32,33], because of their broad substrate specificity [34-36]. Substrates of these pumps are very different in their structural features, while they all tend to have a significant lipophilic moiety [37]. In addition, a single bacterial species can contain multiple homologous RND pumps with overlapping specificities for different antibiotics. The redun- dancy of efflux pumps allows bacteria a compensation of the inhibition or loss of the pump functions with variable expres- sion of efflux components [38,39]. Some can also have a cooperative interaction with other family of drug transporters or act in sequential manner [40-43].

RND transporters cross both the inner and outer membrane forming a tripartite system including the pump located in the cytoplasmic membrane, the outer membrane channel, and the membrane fusion protein located in the periplasm (Figure 1). These three proteins ensure the substrate capture from the outer leaflet of the inner membrane bilayer or the periplasmic space and, the substrate transport across the periplasm and the outer membrane to the external medium receiving energy from the proton motive force $[10,44]$. The three-dimensional structure of the most documented RND pump AcrB of E. coli comprising 12 transmembrane helices was first described as a homotrimeric symmetrical protein [45]. Then crystallographic data (Figure 1) indicate that each subunit exhibits one of the three different conformations [46,47] representing a transport cycle in AcrB. The sequence starts with the loose binding of a substrate in the access pocket (L conformer) to a low affinity site, followed by the tight interaction with the binding pocket ( $\mathrm{T}$ conformer), to finally release the substrate from the opened pocket (O conformer) toward the protein channel TolC. A proton/drug antiport is associated to these steps of substrate translocation. This peristaltic mechanism that involves substrate interactions with the AcrB-binding pocket puts into evidence the pharmacodynamic role of MDR efflux pumps in antibiotic resistance.

To better understand the polyspecificity of efflux pumps and the mode of interaction of substrates, the structure of the large binding pocket of the $\mathrm{T}$ conformer particularly draws the attention of researchers [46]. Polyspecificity means that substrates can interact with different regions of the binding pocket explaining the large diversity of their physicochemical properties. This was demonstrated with the study of the AcrB co-crystallized with minocycline and doxorubicine, two structurally unrelated drugs. The large binding cavity has a

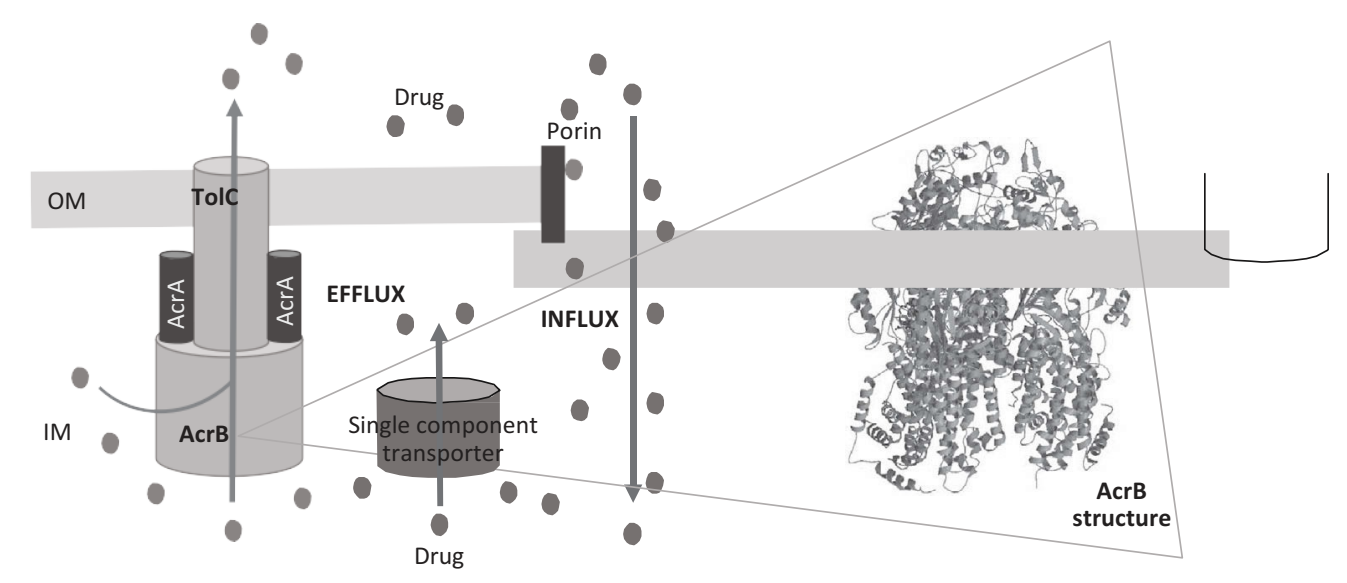

Figure 1. Efflux pumps and pathways of drug influx and efflux across the outer membrane (OM) and the inner membrane (IM) in Gram negative bacteria with details of the AcrB structure of E. coli (2DHH from PDB) [46]. 
hydrophobic area built with 6 phenylalanine residues (such as Phe136, Phe178, Phe610, Phe615, Phe617, and Phe628) and another more polar area with glutamine and asparagine residues (Gln176 and Asn274, respectively). Interactions with these substrates are rather weak. Moreover, AcrB protein contains various hydrophobic areas involving nonspecific binding. Co-crystals with erythromycin and rifampicin have been reported in the access pocket [48]. Finally, the only co-crystal structure with an EPI was obtained with a pyranopyridine substrate [49]. The binding pocket is thus the determining position in the translocation through AcrB. But due to few data available and sufficiently relevant about amino acid residues engaging interactions to identify a workable binding site, understanding this mechanism remains a challenge in the development of new therapeutics aiming at efflux target. So, other methods as computational ones are necessary to study the structure and function of efflux pumps that would allow new drug design.

\section{Efflux, membrane permeability, and intracellular drug concentration}

Changes in membrane permeability allow bacteria to modulate the internal concentration of antibiotics and thus affect the bacterial susceptibility to drugs that most often aim at intracellular targets. Using subinhibitory concentrations of antibacterial agents allows bacteria to trigger other resistance mechanisms via genetic regulation. Porin channels play a direct role in the translocation of polar antibiotics like $\beta$-lactams, whereas hydrophobic biocidal compounds diffuse through the lipid bilayer of inner membrane barrier (Figure 1). Bacteria are able to modify the entrance or the exit of antimicrobial agents by a downregulation of porin production and an overexpression of efflux pumps, respectively. During infections, these bacterial synergistic strategies decrease the antibiotherapy efficacy and thereby ensure dissemination and colonization to the patient. Various gene regulation cascades control membrane permeability. Indeed, several global regulators such as MarA involved in MDR response, strongly affect the expression of porin genes and TolC in Enterobacteriaceae [17,33,50,51]. The transcriptional regulator SoxS is positively controlled by oxidative stress and can trigger MarA expression [26,52]. RamA is an additional global regulator in enteric bacteria (described in Enterobacter, Klebsiella, Salmonella and absent in E. coli) engaged either in the direct control of both porin and efflux expression or through the MarA cascade. This complex regulation mechanism combined with the versatile structure of the outer membrane barrier (i.e. two different lipid bilayers with lipopolysaccharides constituting the external side of the outer membrane) $[18,53]$, governs the translocation of antibacterial agents with different molecular features.

The critical part of the porin channel involved in antibiotic influx is the inner loop 3 determined from porin crystal structures [18]. Alterations in the porin pattern (e.g. in the mutated Omp36 porin) were discovered in clinical resistant isolates as a new mechanism of resistance. Thus, mutations protect the pathogens against antibiotics while maintaining the bacterial survival without reducing the levels of porins for nutrients.
This barrier to the outer membrane permeability is synergistic with the mechanism of resistance involving $\beta$-lactamases that catalyze the hydrolysis of the $\beta$-lactam drugs. However, for hydrophobic antibiotics, the multidrug efflux triggers higher increase of their MIC compared to the low process of drug influx through the bacterial membrane.

Due to their polyspecificity, RND-type efflux systems extrude multiple drugs with very large diversity of structural and physicochemical properties, including ionic, neutral, polar, or hydrophobic molecules with a wide range of steric characters. However, in case of antibiotic classes as aminoglycosides and $\beta$-lactamines that are much less susceptible to AcrB efflux, bacteria take compensatory measures by overexpressing homologous (AcrD) [54,55] efflux components selective for the drugs.

By downregulating influx and upregulating efflux, bacteria control lowers and less effective internal concentrations of toxic agents are available for themselves, while developing resistance processes.

\section{Pumps and pathogenicity}

The active transport of toxic compounds to the outside of the cell allows the survival of bacteria. Nevertheless, RND pumps also have roles in bacterial pathogenicity. Indeed, they are involved in virulence, colonization, production of signaling molecules, and biofilm formation [28,33,56-58]. During infection, bacteria have to overcome the host defense mechanisms to survive. Pathogens employ diverse strategies that include bypassing physical barrier, surviving antimicrobial agents, or producing signals. These strategies contribute to pathogenicity. Clear evidence shows that overexpression of efflux pumps in gram-negative bacteria occurs concurrently with infection $[28,33,58]$.

It was particularly well documented in Salmonella. Deactivation of the binding component of the efflux pump weakens the invasion of tissue culture cells in vitro and colonization in vivo. Indeed, it was shown that two inactivated or deleted efflux pump genes confer to Salmonella a lower ability to adhere to, or invade human intestinal cells compared to single $a \mathrm{cr}$ mutants [58]. And when three of the efflux genes were disabled, adhesion or invasion of Salmonella is almost totally inhibited.

A correlation also exists between RND pumps and the oxidative stress generated during the process of phagocytosis, an important part of the innate immune response during infection. Phagocytic cells produce reactive oxygen species as defense mechanism against bacterial pathogens. As seen in the previous chapter, there is a link between the overexpression RND pumps and oxidative stress mediated by the regulation cascade in the center of which SoxS regulatory system is [26,52].

Inside a bacterial community, gram-negative bacteria use a communication system called quorum sensing that produces and detects signal molecules to coordinate their behavior in a cell-density-dependent manner and control biofilm formation [59]. The extracellular signals mediated by autoinducers allow bacteria to sense the cell density. Indeed, autoinducers trigger a signal production in bacteria that modifies the expression of 
various genes (e.g. autoinducer-dependent genes involved in autoinducer synthesis) in a coordinated response. The most common signaling molecules are $\mathrm{N}$-acyl-homoserine lactones (acyl-HSLs) that can be sensed by specific receptor proteins (LuxR homologues) [60] localized within the cytoplasm or the cell membrane. However, other chemically distinct classes of quorum sensing signal have been discovered including quinolones, boron diesters, and cyclic peptides [61,62]. The quorum sensing messenger system plays many roles in the regulation of bacterial growth, biofilm formation, virulence, and pathogenesis. Indeed, bacteria get used to environmental conditions by selecting related acyl-HSLs among several autoinducers. The length of the acyl side chain (usually from 4 to 18 carbons), saturation and oxidation state, determine the resulting structure of acyl-HSLs and thus their signal specificity. Commonly, acyl-HSLs short chains easily diffuse through membranes whereas with longer ones acyl-HSLs are effluxed. Particularly, it was demonstrated that MexAB-OprM RND pump selects acyl-HSLs and regulates quorum sensing in Pseudomonas aeruginosa [63].

$P$. aeruginosa also produces two quinolone-based signaling molecules: the Pseudomonas quinolone signal (PQS) and the 2heptyl-4(1 H)-quinolone (HHQ), which is a biosynthetic precursor [57].These two quinolone signals bind to the only receptor identified and named PqsR, with a lower affinity in the case of HHQ. PQS is involved in the regulation of several virulence factors and in other bacterial species it influences biofilm formation, bacterial growth, and motility. Affinity probes structurally related to PQS and HHQ have been designed and synthesized in the aim to identified new potential interacting targets from cell lysates of $P$. aeruginosa. PQS affinity probe has successfully captured an inner membrane protein identified as the efflux pump MexG whereas there is no capture from an alkyl quinolone deficient pqsR mutant. The PQS-MexG interactions were also confirmed using purified MexG in vitro and immobilized PQS. It was then shown that this interaction can be out-competed in the presence of ligand in excess. The role of MexG is currently not clear. These experiments show once again that the involvement of efflux systems in virulence and biofilm formation makes RND transporters attractive target for the development of inhibitors.

\section{Efflux pump inhibitors}

The clear evidences on the major role of RND efflux pumps in both antimicrobial resistance and virulence of pathogenic bacteria allow considering the development of new combined therapy using common antibiotics and an efflux pump inhibitor (EPI) as adjuvant $[9,19]$. The behavior of the co-drug consists in no intrinsic antibacterial effect while inducing an increase of intracellular antibiotic concentration and additionally the compound may block the release of virulence factors. Distinct strategies targeting efflux pumps also consider the alteration of pump gene expression, the inhibition of membrane assembly of pump complex, the blocking of the outer membrane exit channel or the collapsing of the energy driven source [64-67]. But the development of inhibitors targeting RND component of efflux pump is now the main approach that is described and tested in gram-negative bacteria. Several series of compounds from both natural $[68,69]$ and synthetic [70-74] sources displaying an EPI activity have been identified (Table 1). Structure-activity relationship (SAR) studies give relevant information about the molecular and the physicochemical properties of EPIs useful in pharmacophore models to describe features that bind the target $[75,76]$. But they often have an incomplete characterization in the various aspects of gram-negative MDR. Crucial studies indicate that, despite their polyspecificity, RND proteins (AcrB or MexB) expel substrates that share identical molecular characteristics. Thus, research efforts are focused to increase affinity of inhibitors to RNDbinding sites related not only to the antibiotic used but also to the homologous RND efflux pump expressed in a selected strain. Since 2002, several 3D-structures of RND proteins have been identified and submitted to the Protein Data Bank (Figure 1). It gives perspectives to increase knowledge about the mechanism of the substrate transport cycle between the three protein conformers and the mode of drug interaction in the ligand-binding sites for EPI-based drug discovery. Nevertheless, the number of EPIs identified by computeraided molecular design associated with mutagenic studies is rather low due to few elucidated pump structures with cocrystallized ligands. Crystallographic structure studies give significant results but are not sufficient to understand all structural aspects of ligand interaction-based drug design. Currently, despite progress in crystallogenesis, it remains difficult to obtain co-crystals.

Computational methods help understanding the relationship between the structure and the function of the protein under study by simulating molecular interactions and conformational changes of a model built from its 3D-structure and sequence homology. High-resolution 3D-structures make it achievable. Using rigid docking, it is possible to identify most energetically favorable poses of small molecules such as EPIs in a specific binding site. Diverse computational studies using virtual screening of broad compound databases [22] can assist the determination of the EPI mode of action analyzing how they bind to the efflux target at the molecular level. As an example, a chemical library of 30 compounds among which minocycline, doxorubicin, tetracycline, and levofloxacin has been virtually screened on a AcrB model of E. coli [70]. It was shown that compounds bind two sites called the 'groove' and the 'cave' regions. This docking study was supported by biochemical data from cell-based efflux pump activity assays. Molecular dynamics (MD) simulations are complementary techniques to elucidate functional biological targets. MD allows to estimate the contribution of the amino acid residues involved in the interaction with the ligand. This method confers a more realistic model of the binding site compared to the rigid docking. Indeed, it includes the flexibility of the binding site while taking into account the presence of water molecules. Using this method, the interaction of known structurally unrelated substrates of AcrB (such as minocycline, taurocholic acid, nitrocefin, chloramphenicol, ethidium, oxacillin, ciprofloxacin, cephalotin, and erythromycin), the two EPIs (Table 1) phenylalanyl-arginine- $\beta$ naphthylamide $(\mathrm{PA} \beta \mathrm{N})$ and 1-(1-naphthylmethyl)-piperazine (NMP) and the two non-substrates (such as kanamycin A and glucose) have been analyzed [71] in the distal binding pocket 
Table 1. Chemical series from natural $(\mathrm{N})$ and synthetic (S) origin displaying EPI activity associated to antibiotics and bacterial species (updated from the previously published data in reference [19]).

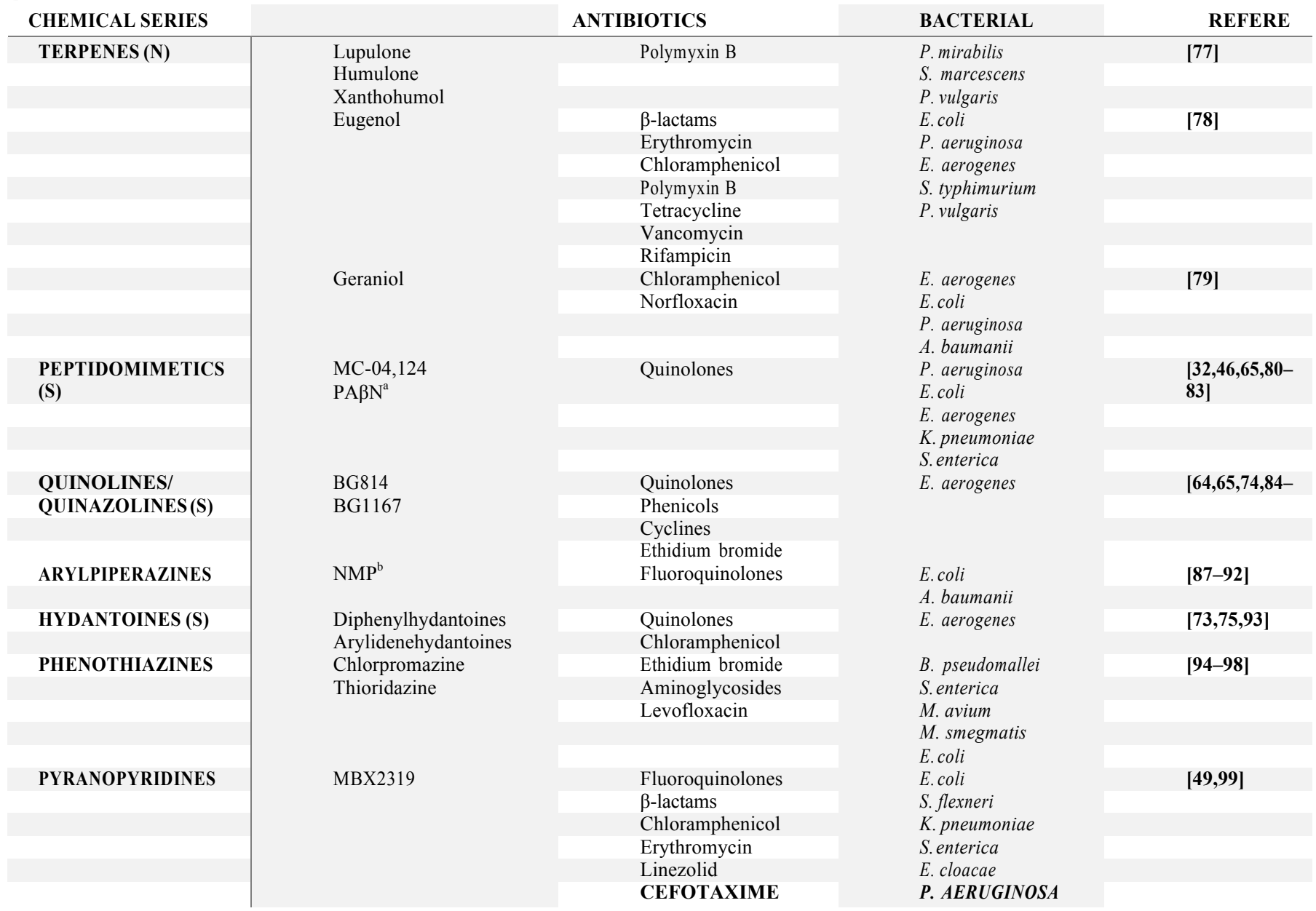

${ }^{a}$ Phenylalanyl-arginine- $\beta$-naphthylamide.

${ }^{\mathrm{b}} 1$-(1-naphthylmethyl)-piperazine.

(identified by X-ray crystallography) in terms of the binding energy, hydrophobic surface matching, and the residues involved in the process. It was observed that only efflux substrates bound the distal pocket while EPIs preferred binding a glycine-rich loop known to control the access of substrates to the binding sites. As the predictive accuracy of these methods continuously increases, molecular modeling is the most promising approach to design EPI.

EPIs can be regarded as new antibacterial agents developed to bypass antibiotic resistance restoring drug concentration $[65,80]$ inside the bacteria cell by blocking efflux pump activity. Among them, competitive inhibitors of antibiotic translocation through AcrB have been reported [64]. Only few studies evaluated EPIs in clinical isolates (e.g. E. coli, E. aerogenes, $K$. pneumoniae, or $P$. aeruginosa) expressing efflux pumps. As antibiotic drugs, the key step of EPIs effect is their uptake through the outer membrane of bacteria [65]. EPIs activity is also notably dependent on the class of the antibiotic to which they are combined thus resulting in discrepancies between the activity level of antibiotic restored and the EPIs concentration used. This was examined in different comparative studies using quinoline/ quinazoline derivatives and $\mathrm{PA} \beta \mathrm{N}$ as a standard $[64,65,74,84-86]$. It was observed that they do not have the same chemosensitizing effect on ciprofloxacin, sparfloxacin, and erythromycin activity in E. aerogenes bacteria overexpressing AcrB efflux pump. The different affinity of antibiotic and EPI ligands for the efflux binding sites could influence their respective activity associated with the level and the nature of efflux pump expressed in a given strain and the conditions used in screening assays. Comparable results have been obtained between PA $\beta N$ and NMP inhibitors [86] in E. coli, especially observed with the macrolide class of antibiotics. There is a strong relationship linking the specificity of efflux pump and the efficacy of EPIs which thus implies selecting the suitable drug design strategy. Actually, in this context ligand-based drug design seems the most promising approach.

Finally, a clinically applicable EPI must fill in standard pharmacodynamic features [80] as: (i) increasing the susceptibility to antibiotics of bacterial-resistant strains expressing efflux pumps, (ii) action on susceptible strains deleted of specific drug efflux pumps, (iii) no pharmacological effect on eukaryotic targets, (iv) no potentiation activity on not expelled antibiotics, (v) increasing the intra-bacterial concentration of efflux pump substrates, (vi) decreasing the extrusion level of efflux pump substrates, (vii) no impact on the membrane proton gradient. 


\section{Conclusion}

It was shown that antibiotic efflux is the first line of bacterial defense against these drugs conferring only low level of resistance but allowing as a result the development of additional resistance mechanisms that lead to higher degree of resistance. Efflux proteins belonging to the RND family play an important role in the intrinsic resistance of gram-negative bacteria. But it is clear that antibiotic efflux is not their first physiological function. Understanding their original functions and the conditions that promote their expression would allow identifying the way to bypass MDR mediated by these pumps. This knowledge would be useful to predict the appearance of MDR phenotypes in clinic and could improve efficacy of treatment settings. The expression of efflux pumps is strongly regulating by a complex balance between local and global regulators. Diverse stress conditions mediated by global regulatory mechanisms also cause the overexpression of efflux pumps contributing to MDR phenotype. The broad specificity of RND pumps confers to bacteria exposed to one efflux substrate the capacity to develop cross-resistance to multiple antibiotics having different physicochemical properties. This polyspecificity is also an obstacle to the discovery and the design of new drugs. Nevertheless, clear evidence shows that aiming MDR gram-negative bacteria efflux pumps is a crucial strategy to develop new treatments to circumvent antibiotic/ antiseptic resistance and virulence by(i) decreasing intrinsic and acquired antibiotic resistance of bacteria, (ii) reversing the development of additional mechanisms of resistance, (iii) reaching an effective intracellular concentration capable to inhibit bacterial growth, (iv) inhibiting the release of virulence factors associated to the capacity of bacterial adhesion and invasion.

The lack of new antibiotic therapies and the widespread of bacterial resistance to multiple drugs enlighten the urgent need to identify and validate new targets. Original innovative pharmacodynamic perspectives in research and development are required for producing anti-infective drugs. Development of EPIs against RND efflux target as adjuvant of antibiotics would allow recovering, at least partly, the golden age of antibacterial agents for some antibiotic families.

\section{Expert opinion}

As the alteration of the efflux functions in bacteria modulate the expression of homologous RND pumps, this bacterial compensatory system must be taken into account to develop selective EPIs not only regarding the molecular structure of the expelled antibiotic but also the one of the RND efflux component. Indeed, on a one hand EPIs are able to potentiate the activity of some antibiotic classes but on the other hand EPIs decrease bacterial susceptibility to some others as in the case of aminoglycosides [58]. Conversely, as acyl-HSLs virulence factors are selective regarding efflux pumps to be released in the extracellular medium, the use of an EPI to inhibit multiple pumps in this case would allow obtaining an additive effect to reduce pathogenicity of bacteria. Improving selectivity would also allow decreasing adverse effects of EPIs that contributed till now to low progress in preclinical or clinical trials. The multipurpose of an EPI that inhibit all functions of efflux pumps is thus in question: choosing additive effect or selective effect of an EPI must consider the impact of one or the other strategy on the subsequent pressure of selection on the bacterial population and thus on the emergence of resistance. So, advances in crystallography, in molecular modeling, and in dynamics simulations would allow a better determination of how EPIs bind to the efflux site in order to improve their efficacy as adjuvant for a given antibiotic structural class. Moreover, this opens the way for a diagnostic tool detecting an important efflux phenotype in clinical isolates and thus facilitating the treatment adaptation to combat the bacterium. Computer-aided drug design is a step in the way from drug candidate discovery to drug development. The finding of hit compounds can use two different approaches of virtual screening: one based on the ligand structure another based on molecular fragments. From these two approaches, structurally unrelated EPIs have been discovered in the last decade: ones are analogs of antibiotic substrates sharing the same scaffold and the same structural features interacting with the efflux transporter but not those involved in the binding to the antibacterial target; and others having new distinct scaffolds. One of the advantages to use antibiotic analogs is preserving the available druggability parameters of the substance compared with those for which no pharmacokinetic data exist. But the major drawback to overcome is producing analogs sufficiently selective regarding homologous efflux pumps and also regarding antibacterial target that have no intrinsic antibacterial effect while inducing an increase of intracellular antibiotic concentration. Another key challenge is the capacity to quantify in real time the antibiotic concentration required inside the bacterium to bring back an optimal antibacterial activity in the presence of an EPI adjuvant. Commonly, we use minimal inhibitory concentration (MIC) measurements to evaluate drug efflux comparing the activity in bacteria expressing differently efflux in the presence or in the absence of EPI, and radiolabeled substrates to quantify their accumulation inside bacteria. But SAR studies cannot be refined with MIC approach as only wide differences in MIC values allow to determine significant effects. Moreover, this is often difficult to find relationship between MIC values and direct efflux measurements using fluorescent dyes substrate of efflux (e. g. ethidium bromide, 1,2' -dinaphthylamine, Hoechst H33342). Advances in new noninvasive methodologies using fluorescence spectrometry associated to cell imaging by microscopy allow detecting and quantifying drugs inside individual living bacteria [100]. The significant obstacle to these methods is the interfering fluorescence of molecules used in the evaluation. The development of quantitative mass spectroscopy of compounds with any physicochemical properties can help in solving this aspect. All these techniques quantify efflux activity directly usable to refine SAR studies and rationalize the design of EPIs.

\section{Acknowledgments}

We appreciate greatly fruitful discussions and advices from Anne DavinRegli and Jean-Michel Bolla.

\section{Funding}

This work was supported by grant ANR-11-BS07-019-01 'IBEF' Agence Nationale de la Recherche (ANR, France) and partly supported by AixMarseille University and IRBA. 


\section{Declaration of interest}

The authors have no other relevant affiliations or financial involvement with any organization or entity with a financial interest in or financial conflict with the subject matter or materials discussed in the manuscript apart from those disclosed.

\section{References}

1. Antimicrobial Resistance: Global Report on Surveillance 2014. WHO. 2014. Available from: http://www.who.int/drugresistance/docu ments/surveillancereport/en/

2. Blot S, Depuydt P, Vandewoude K, et al. Measuring the impact of multidrug resistance in nosocomial infection. Curr Opin Infect Dis. 2007;20:391-396.

3. Falagas ME, Bliziotis IA. Pandrug-resistant Gram-negative bacteria: the dawn of the post-antibiotic era? Int J Antimicrob Agents. 2007;29:630636.

4. Chopra I, Schofield C, Everett M, et al. Treatment of health- careassociated infections caused by Gram-negative bacteria: a consen- sus statement. Lancet Infect Dis. 2008;8:133-139.

5. Rice LB. The clinical consequences of antimicrobial resistance. Curr Opin Microbiol. 2009;12:476-481.

6. Gandhi TN, DePestel DD, Collins CD, et al. Managing antimicrobial resistance in intensive care units. Crit Care Med. 2010;38(Suppl 8): S315-23.

7. Boucher HW, Talbot GH, Bradley JS, et al. Bad bugs, no drugs: no ESKAPE! An update from the infectious diseases society of America. Clin Infect Dis. 2009;48:1-12.

8. Rice LB. Progress and challenges in implementing the research on ESKAPE pathogens. Infect Control Hosp Epidemiol. 2010;31(Supp1 1):S7-S10.

9. Nikaido H, Pagès J-M. Broad-specificity efflux pumps and their role in multidrug resistance of Gram-negative bacteria. FEMS Microbiol Rev. 2012;36:340-363.

10. Li ZH, Plesiat P, Nikaido H. The challenge of efflux-mediated antibiotic resistance in Gram-negative bacteria. Clin Micro Rev. 2015;28:337-418.

11. Tumah HN. Bacterial biocide resistance. J Chemother. 2009;21:5-15.

12. Hegstad K, Langsrud S, Lunestad BT, et al. Does the wide use of quaternary ammonium compounds enhance the selection and spread of antimicrobiol resistance and thus threaten our health ? Microb Drug Resist. 2010;16:91-104.

13. Delmar JA, Su -C-C, Yu EW. Bacterial multidrug efflux transporters. Annu Rev Biophys. 2014;43:93-117.

14. Blair JM, Webber MA, Baylay AJ, et al. Molecular mechanisms of antibiotic resistance. Nat Rev Microbiol. 2015;13:42-51.

15. Venter H, Mowla R, Ohene-Agyei T, et al. RND-type drug efflux pumps from Gram-negative bacteria: molecular mechanism and inhibition. Front Microbiol. 2015;6:377.

16. Davin-Regli A, Masi M, Bialek S, et al. Antimicrobial resistance and drug efflux pumps in Enterobacter and Klebsiella. In: Li X-Z, Elkins CA, Zgurskaya HI, editors. Efflux-mediated drug resistance in bac- teria: Mechanisms, regulation and clinical implications. Berlin: Springer; 2016.

17. Davin-Regli A, Bolla JM, James CE, et al. Membrane permeability and regulation of drug 'influx and efflux' in enterobacterial patho- gens. Curr Drug Targets. 2008;9:750-759.

-. Understanding the relationships between influx and efflux mechanisms is crucial to overcome antibiotic resistance.

18. Pagès JM, James CE, Winterhalter $\mathrm{M}$. The porin and the permeating antibiotic: a selective diffusion barrier in Gram-negative bacteria. Nat Rev Microbiol. 2008;6:893-903.

19. Bolla JM, Alibert-Franco S, Handzlik J, et al. Strategies for bypassing the membrane barrier in multidrug resistant Gram-negative bac- teria. FEBS Lett. 2011;585:1682-1690.
20. Ruggerone P, Murakami S, Pos KM, et al. RND efflux pumps: structural information translated into function and inhibition mechanisms. Curr Top Med Chem. 2013;13:3079-3100.

21. Dreier J, Ruggerone P. Interaction of antibacterial compounds with RND efflux pumps in Pseudomonas aeruginosa. Front Microbiol. 2015;6:660.

22. Opperman TJ, Nguyen ST. Recent advances toward a molecular mechanism of efflux pump inhibition. Front Microbiol. 2015;6:421.

23. Jones D. News and analysis: the antibacterial lead discovery challenge. Nat Rev Drug Discov. 2010;9:751-752.

24. Yamaguchi A, Nakashima R, Sakurai K. Structural basis of RND-type multidrug exporters. Front Microbiol. 2015;6:327.

25. Poole K, Krebes K, McNally C, et al. Multiple antibiotic resistance in Pseudomonas aeruginosa: evidence for involvement of an efflux operon. J Bacteriol. 1993;175:7363-7372.

26. Ma D, Cook DN, Alberti M, et al. Genes acrA and acrB encode a stress-induced efflux system of Escherichia coli. Mol Microbiol. 1995; 16:45-55.

27. Du D, Voss J, Wang Z, et al. The pseudo-atomic structure of an RND-type tripartite multidrug efflux pump. Biol Chem. 2015;396:1073-1082.

28. Blair JMA, Piddock LJV. Structure, function and inhibition of RND efflux pumps in Gram-negative bacteria: an update. Curr Opin Microbiol. 2009;12:512-519.

29. Ogawa W, Onishi M, Ni R, et al. Functional study of the novel multidrug efflux pump KexD from Klebsiella pneumoniae. Gene. 2012;498:177-182.

30. Nowak J, Seifert H, Higgins PG. Prevalence of eight resistancenodulation-division efflux pump genes in epidemiologically characterized Acinetobacter baumannii of worldwide origin. J Med Microbiol. 2015;64:630-635.

31. Tsukagoshi N, Aono R. Entry into and release of solvents by Escherichia coli in an organic-aqueous two-liquid-phase system and substrate specificity of the AcrABTolC solvent-extruding pump. J Bacteriol. 2000;182:4803-4810.

32. Lomovskaya O, Zgurskaya HI, Totrov M, et al. Waltzing transporters and 'the dance macabre' between humans and bacteria. Nat Rev Drug Discov. 2007;6:56-65.

33. Piddock LJ. Multidrug-resistance efflux pumps - not just for resistance. Nat Rev Microbiol. 2006;4:629-636.

34. Poole K. Efflux-mediated antimicrobial resistance. J Antimicrob Chemother. 2005;56:20-51.

35. Elkins CA, Nikaido H. 3D structure of AcrB: the archetypal multidrug efflux transporter of Escherichia coli likely captures substrates from periplasm. Drug Resist Update. 2003;6:9-13.

36. Pradel E, Pages J-M. The AcrAB-TolC pump contributes to multidrug resistance in the nosocomial pathogen Enterobacter aerogenes. Antimicrob Agents Chemother. 2002;46:2640-2643.

37. Nikaido $H$. Multidrug efflux pumps of gram-negative bacteria. J Bacteriol. 1996;178:5853-5859.

38. Eaves DJ, Ricci V, Piddock LJV. Expression of acrB, acrF, acrD, marA and soxS in Salmonella enterica serovar Typhimurium: role in multiple antibiotic resistance. Antimicrob Agents Chemother. 2004;48:1145-1150.

39. Biot FV, Lopez MM, Poyot T, et al. Interplay between RND efflux pumps in doxycycline-selected strains of Burkholderia thailandensis. Plos One. 2013;8:e84068.

- The redundancy of efflux pumps highlights the problem of polyspecificity of the RND target.

40. Lee A, Mao W, Warren MS, et al. Interplay between efflux pumps may provide either additive or multiplicative effects on drug resistance. J Bacteriol. 2000;182:3142-3150.

41. Tal N, Schuldiner S. A coordinated network of transporters with overlapping specificities provides a robust survival strategy. P Natl Acad Sci USA. 2009;106:9051-9056.

-. The cooperative interaction between drug transporters emphasizes their importance in antibiotic resistance.

42. Hobbs EC, Yin X, Paul BJ, et al. Conserved small protein associates with the multidrug efflux pump AcrB and differentially affects antibiotic resistance. P Natl Acad Sci USA. 2012;109:16696-16701. 
43. Du D, Wang Z, James NR, et al. Structure of the AcrAB-TolC multidrug efflux pump. Nature. 2014;509:512-515.

44. Shuster Y, Steiner-Mordoch S, Alon-Cudkowicz N, et al. A transpor- ter interactome is essential for the acquisition of antimicrobial resitance to antibiotics. Plos One. 2016;11:e0152917.

45. Murakami S, Nakashima R, Yamashita E, et al. Crystal structure of bacterial efflux transporter AcrB. Nature. 2002;419:587-593.

46. Murakami S, Nakashima R, Yamashita E, et al. Crystal structures of a multidrug transporter reveal a functionnally rotating mechanism. Nature. 2006;443:173-179.

47. Seeger MA, Schiefner A, Eicher T, et al. Structural asymmetry of AcrB trimer suggests a peristaltic pump mechanism. Science. 2006;313:1295-1298.

48. Nakashima R, Sakurai K, Yamasaki S, et al. Structures of the multidrug exporter AcrB reveal a proximal multisite drug binding pocket. Nature. 2011;480:565-569.

49. Sjuts H, Vargiu AV, Kwasny SM, et al. Molecular basis for inhibition of AcrB multidrug efflux pump by novel and powerful pyranopyr- idine derivatives. P Natl Acad Sci USA. 2016;113:3509-3514.

50. Levy SB. Active efflux, a common mechanism for biocide and antibiotic resistance. Sym Ser Soc Appl Microbiol. 2002;92:65S-71S.

51. Alekshun MN, Levy SB. Molecular mechanisms of antibacterial multidrug resistance. Cell. 2007;128:1037-1050.

52. Masi M, Pagès J-M. Structure, function and regulation of outer membrane proteins involved in drug transport in Enterobacteriaceae: the OmpF/C-TolC case. Open Microbiol J. 2013;7:22-33.

53. Nikaido H. Molecular basis of bacterial outer membrane permeabil- ity revisited. Microbiol Mol Biol Rev. 2003;67:593-656.

54. Rosenberg EY, Ma D, Nikaido H. AcrD of Escherichia coli is an aminoglycoside efflux pump. J Bacteriol. 2000;182:1754-1756.

55. Aires JR, Nikaido H. Aminoglycosides are captured from both periplasm and cytoplasm by the AcrD multidrug efflux transporter of Escherichia coli. J Bacteriol. 2005;187:1923-1929.

56. Baugh S, Piddock LJV. Salmonella efflux pumps. Microbial Efflux Pumps. 2013;163-173.

57. Hodgkinson JT, Gross J, Baker YR, et al. A new pseudomonas quinolone signal (PQS) binding partner: MexG. Chem Sci. 2016;7:2553-2562.

58. Blair JMA, Smith HE, Ricci V, et al. Expression of homologous RND efflux pump genes is dependent upon AcrAB expression: implications for efflux and virulence inhibitor design. J Antimicrob Chemother. 2015;70:424-431.

59. Waters CM, Bassler BL. Quorum sensing: cell-to-cell communication in bacteria. Ann Rev Cell Developmental Biol. 2005;21:319-346.

60. Galloway WRDJ, Hodgkinson JT, Bowden SD, et al. Quorum sensing in Gram-negative bacteria: small molecule modulation of AHL and AI-2 quorum sensing pathways. Chem Rev. 2011;111:28-67.

61. Atkinson S, Williams P. Quorum sensing and social networking in the microbial world. J R Soc Interface. 2009;6:959-978.

62. Antunes LCM, Ferreira RBR, Buckner MMC, et al. Quorum sensing in bacterial virulence. Microbiol. 2010;156:2271-2282.

63. Minagawa $\mathrm{S}$, Inami $\mathrm{H}$, Kato $\mathrm{T}$, et al. RND type efflux system MexABOprM of Pseudomonas aeruginosa selects bacterial languages, 3- oxoacyl-homoserine lactones, for cell-to-cell communication. BMC Microbiol. 2012;12:70.

64. Pagès J-M, Alibert-Franco S, Mahamoud A, et al. Efflux pumps of gram-negative bacteria, a new target for new molecules. Curr Top Med Chem. 2010;8:1848-1857.

65. Pagès J-M, Amaral L. Mechanisms of drug efflux and strategies to combat them: challenging the efflux pump of Gram-negative bacteria. Biochim Biophys Acta. 2009;1794:826-833.

66. Falagas ME, Grammatikos AP, Michalopoulos A. Potential of oldgeneration antibiotics to address current need for new antibiotics. Expert Rev Anti Infect Ther. 2008;6:593-600.

67. Yeaman MR, Yount NY. Mechanisms of antimicrobial peptide action and resistance. Pharmacol Rev. 2003;55:27-55.

68. Ohene-Agyei T, Mowla R, Rahman T, et al. Phytochemicals increase the antibacterial activity of antibiotics by acting on a drug efflux pump. Microbiol Open. 2014;3:885-896.
69. Aparna V, Dineshkumar K, Mohanalakshmi N, et al. Identification of natural compound inhibitors for multidrug efflux pumps of Escherichia coli and Pseudomonas aeruginosa using in silico highthroughput virtual screening and in vitro validation. Plos One. 2014;9:e101840.

70. Takatsuka Y, Chen C, Nikaido H. Mechanism of recognition of compounds of diverse structures by the multidrug efflux pump AcrB of Escherichia coli. P Natl Acad Sci USA. 2010;107:6559-6565.

71. Vargiu AV, Nikaido H. Multidrug binding properties of the AcrB efflux pump characterized by molecular dynamics simulations. $P$ Natl Acad Sci USA. 2012;109:20637-20642.

72. Nguyen ST, Kwasny SM, Ding X, et al. Structure-activity relationships of a novel pyranopyridine series of Gram-negative bacterial efflux pump inhibitors. Bioorg Med Chem. 2015;23:2024-2034.

73. Otrebska-Machaj E, Chevalier J, Handzlik J, et al. Efflux pump blockers in Gram-negative bacteria: the new generation of hydantoin based modulators to improve antibiotic activity. Front Microbiol. 2016;7:622.

74. Mahamoud A, Chevalier J, Baitiche M, et al. An alkylaminoquinazoline restores antibiotic activity in Gram-negative resistant isolates. Microbiol. 2011;157:566-571.

75. Handzlik J, Szymańska E, Alibert S, et al. Search for new tools to combat Gram-negative resistant bacteria among amine derivatives of 5-arylidenehydantoin. Bioorg Med Chem. 2013;21:135-145.

- Description of important molecular features of EPIs using a pharmacophore model.

76. Nakayama K, Kawato H, Watanabe J, et al. MexAB-OprM specific efflux pump inhibitors in Pseudomonas aeruginosa. Part 3: optimization of potency in the pyridopyrimidine series through the application of a pharmacophore model. Bioorg Med Chem Lett. 2004;14:475-479.

- Molecular characteristics of EPIs highlight their mode of interaction with efflux pumps.

77. Natarajan P, Katta S, Andrei I, et al. Positive antibacterial co-action between hop (Humulus lupulus) constituents and selected antibiotics. Phytomed. 2008;15:195-201.

78. Hemaiswarya S, Doble M. Synergistic interaction of eugenol with antibiotics against Gram negative bacteria. Phytomed. 2009;16:9971005.

79. Lorenzi V, Muselli A, Bernardini AF, et al. Geraniol restores antibiotic activities against multidrug resistant isolates from Gram negative species. Antimicrob Agents Chemother. 2009;53:2209-2211.

80. Lomovskaya O, Bostian KA. Practical applications and feasibility of efflux pump inhibitors in the clinic - a vision for applied use. Biochemical Pharmacology. 2006;71:910-918.

- The pharmacodynamic role of efflux pumps and the pharmacodynamic features of EPIs.

81. Watkins WJ, Landaverry Y, Leger R, et al. The relationship between physicochemical properties; in vitro activity and pharmacokinetic profile of analogues of diamine-containing efflux pump inhibitors. Bioorg Med Chem Lett. 2003;13:4241-4244.

82. Kriengkauykiat J, Porter E, Lomovskaya O, et al. Use of an efflux pump inhibitor to determine the prevalence of efflux pumpmediated fluoroquinolone resistance and multidrug resistance in Pseudomonas aeruginosa. Antimicrob Agents Chemother. 2005;49:565-570.

83. Yoshida K, Nakayama K, Ohtsuka M, et al. MexAB-OprM specific efflux pump inhibitors in Pseudomonas aeruginosa;Part 7: highly soluble and in vivo active quaternary ammonium analogue D139001: a potential preclinical candidate. Bioorg Med Chem. 2007;15:7087-7097.

84. Chevalier J, Mahamoud A, Baitiche M, et al. Quinazoline derivatives are efficient chemosensitizers of antibiotic activity in Enterobacter aerogenes, Klebsiella pneumoniae and Pseudomonas aeruginosa resistant strains. Int J Antimicrob Agents. 2010;36:164-168.

85. Mahamoud A, Chevalier J, Alibert-Franco S, et al. Antibiotic efflux pumps in Gram-negative bacteria: the inhibitor response strategy. J Antimicrob Chemother. 2007;59:1223-1229.

86. Mahamoud A, Chevalier J, Baitiche M, et al. An alkylaminoquinazoline restores antibiotic activity in Gram negative resistant isolates. Microbiol 2011;157:566-571. 
87. Bohnert JA, Kern WV. Selective arylpiperazines are capable of reversing multidrug resistance in Escherichia coli overexpressing RND efflux pumps. Antimicrob Agents Chemother. 2005;49:849-852.

88. Kern WV, Steinke P, Schumacher A, et al. Effect of 1-(1-naphthylmethyl)-piperazine, a novel putative efflux pump inhibitor, on antimicrobial drug susceptibility in clinical isolates of Escherichia coli. J Antimicrob Chemother. 2006;57:339-343.

89. Schumacher A, Steinke P, Bohnert JA, et al. Effect of 1-(1-naphthylmethyl)-piperazine, a novel putative efflux pump inhibitor, on antimicrobial drug susceptibility in clinical isolates of Enterobacteriaceae other than Escherichia coli. J Antimicrob Chemother. 2006;57:344-348.

90. Pannek S, Higgings PG, Steinke P, et al. Multidrug efflux inhibition in Acinetobacter baumanii: comparison between 1-(1-naphthylmethyl)-piperazine and phenyl-arginine-beta-naphthylamide. J Antimicrob Chemother. 2006;57:9704.

91. Schumacher A, Trittler R, Bohnert JA, et al. Intracellular accumulation of linezolid in Escherichia coli, Citrobacter freundii and Enterobacter aerogenes: role of enhanced efflux pump activity and inactivation. J Antimicrob Chemother. 2007;59:1261-1264.

92. Hannula M, Hänninen ML. Effect of putative efflux pump inhibitors and inducers on the antimicrobial susceptibility of Campilobacter jejuni and Campilobacter coli. J Med Microbiol. 2008;57:851-855.

93. Handzlik J, Szymanska E, Chevalier J, et al. Amine-alkyl derivatives of hydantoin: new tool to combat resistant bacteria. Eur J Med Chem. 2011;46:5807-5816.
94. Martins M, Dastidar SG, Fanning S, et al. Potential role of nonantibiotics (helper compounds) in the treatment of multidrug-resistant Gram-negative infections: mechanisms for their direct and indirect activities. Int J Antimicrob Agents. 2008;31:198-208.

95. Bailey AM, Paulsen IT, Piddock LJ. RamA confers multidrug resistance in Salmonella enterica via increased expression of acrB; which is inhibited by chlorpromazine. Antimicrob Agents Chemother. 2008;52:3604-3611.

96. Rodrigues L, Wagner D, Viveiros M, et al. Thioridazine and chlorpromazine inhibition of ethidium bromide efflux in Mycobacterium avium and Mycobacterium smegmatis. J Antimicrob Chemother. 2008;61:1076-1082.

97. Amaral L, Martins M, Viveiros M, et al. Promising therapy of XDR-TB/ MDR-TB with thioridazine an inhibitor of bacterial efflux pumps. Curr Drug Targets. 2008;9:816-819.

98. Viveiros M, Martins M, Couto I, et al. New methods for the identification of efflux mediated MDR bacteria; genetic assessment of regulators and efflux pump constituents; characterization of efflux systems and screening for inhibitors of efflux pumps. Curr Drug Targets. 2008;9:760-778.

99. Aron Z, Opperman TJ. Optimisation of a novel series of pyranopyridine RND efflux pump inhibitors. Curr Opin Microbiol. 2016;33:1-6.

100. Cinquin B, Maigre L, Pinet E, et al. Microspectrometric insights on the uptake of antibiotics at the single bacterial cell level. Sci Rep. 2015;5:17968 